\title{
DPPA2 Protein Expression is Associated with Gastric Cancer Metastasis
}

\author{
Hoda Shabestarian ${ }^{1 \&}$, Mohammad Ghodsi ${ }^{2 \&}$, Afsaneh Javdani Mallak ${ }^{3 \&}$, Amir \\ Hossein Jafarian ${ }^{4}$, Mehdi Montazer ${ }^{5}$, Mohammad Mahdi Forghanifard ${ }^{6 *}$
}

\begin{abstract}
Gastric cancer (GC) as the fourth most common cause of malignancies shows high rate of morbidity appropriating the second leading cause of cancer-related death worldwide. Developmental pluripotency associated-2 (DPPA2), cancer-testis antigen (CT100), is commonly expressed only in the human germ line and pluripotent embryonic cells but it is also present in a significant subset of malignant tumors. To investigate whether or not DPPA2 expression is recalled in GC, our aim in this study was to elucidate DPPA2 protein expression in gastric cancer. Fifty five GC tumor and their related margin normal tissues were recruited to evaluate DPPA2 protein expression and its probable associations with different clinicopathological features of the patients. DPPA2 was overexpressed in GC cases compared with normal tissues $(\mathbf{P}<.005)$. While DPPA2 expression was detected in all GC samples, its high expression was found in 23 of 55 tumor tissues (41.8\%). Interestingly, 50 of 55 normal samples $(\mathbf{9 0 . 9 \%})$ were negative for DPPA2 protein expression and remained 5 samples showed very low expression of DPPA2. DPPA2 protein expression in GC was significantly correlated with lymph node metastasis $(p=0.012)$. The clinical relevance of DPPA2 in GC illustrated that high level expression of this protein was associated with lymph node metastasis supporting this hypothesis that alteration in DPPA2 was associated with aggressiveness of gastric cancer and may be an early event in progression of the disease. DPPA2 may be introduced as a new marker for invasive and metastatic GCs.
\end{abstract}

Keywords: Gastric cancer - DPPA2 - immunohistochemistry - metastasis

Asian Pac J Cancer Prev, 16 (18), 8461-8465

\section{Introduction}

Gastric cancer (GC) as the fourth most common cause of malignancies shows high rate of morbidity appropriating the second leading cause of cancer-related death worldwide (Mottaghi-Dastjerdi et al., 2014; Wu et al., 2014). The considerable geographical variations have been reported for GC distribution. While GC incidence rate in developed regions such as Norway is very low (6.9 and3.0 per 105 in males and females, respectively) (Hudler, 2012), in less developed areas, such as Eastern Asia, it is approximately 6-fold higher (42.4 and $18.3 \mathrm{vs}$. 6.9 and 3.0) (Ferlay et al., 2010).

Among gastrointestinal carcinomas, gastric adenocarcinoma is genetically, cytologically and architecturally heterogeneous. Therefore, investigating trusty tumor markers and searching for prognostic indicators is difficult. Although there are many studies focusing on single or multiple genes for good prediction of GC (Takeno et al., 2010; Cho et al., 2011), no gene signature or any biomarkers is introduced in conventional clinical prognosis. Indeed, understanding the mechanisms and complications leading to GC is one of the major challenges in cancer genomics.

Embryo-cancer sequence A (ECSA), a member of cancer-embryo antigens or developmentally restricted differentiation antigens (DRDAGs), is entered into the gene databases as developmental pluripotency associated-2 (DPPA2) which is also recognized as cancer-testis antigens (CTs) named CT100. This protein is commonly expressed only in the human germ line and pluripotent embryonic cells but it is also present in a significant subset of malignant tumors. CT100 antigen may prove to be a marker of 'repopulating' cells with stem cell-like characteristics (Tchabo et al., 2009). ECSA/ DPPA2 gene maps to chromosome 3 q13 over 9 exons and encodes a protein product of 297 amino acids. The primary protein structure contains a SAP (SAF-A/B, Acinux and PIAS) motif and localizes into the nucleus (John et al., 2008). DPPA 2 comprised of a common purported DNAbinding domain, the SAP motif, which has DNA/RNAbinding ability and is pertained to chromatin modification

${ }^{1}$ Department of Basic Sciences, Faculty of Veterinary Medicine, Ferdowsi University of Mashhad, ${ }^{2}$ Cancer Molecular Pathology Research Center, Faculty of Medicine, ${ }^{3}$ Division of Human Genetics, Immunology Research Center, Avicenna Research Institute, ${ }^{4}$ Department of Pathology, Ghaem Hospital, Mashhad University of Medical Sciences, Mashhad, ${ }^{5}$ Department of pathology, EmamKhomeini Hospital, Ilam University of Medical Sciences, Ilam, ${ }^{6}$ Department of Biology, Damghan Branch, Islamic Azad University, Damghan, Iran ${ }^{\circledR}$ Equal contributors *For correspondence: forghanifard@gmail.com 
Hoda Shabestarian et al

(Aravind and Koonin, 2000). The ubiquitous expression of Dppa2 is observed in germinal vesicle stage oocytes and during the cleavage stages of mouse embryogenesis. Dppa2 expression is restrained to the inner cell mass of blastocysts where embryonic stem cells are developing. In the course of embryogenesis, Dppa2 is expressed only in the developing germ line, suggesting that Dppa2 expression is similar to expression of pluripotent markers and targets a subset of pluripotent cells (MaldonadoSaldivia et al., 2007). Moreover, the under expression of Dppa2 and other pluripotent markers such as OCT4 and NANOG is showed during differentiation of mouse embryonic stem cells (Du et al., 2010). DPPA2, as the human homolog of Dppa2, was reported to be coexpressed with OCT4 in human ES cells, blastocysts and primordial germ cells (Monk et al., 2008), which shows evolutionally conserved expression of Dppa2.

GC is derived by three known molecular mechanisms including chromosome instability, microsatellite instability and epigenetic alterations (Tan et al., 2012), which lead to deregulation of critical genes expression and cell signaling pathways, thoroughly (Hudler, 2012; Zabaleta, 2012). To investigate whether or not DPPA2 expression is recalled in GC, our aim in this study was to determine DPPA2 protein expression in tumor tissues of GC and their related margin normal tissues to evaluate the probable associations between the protein expression and different clinicopathological variables of the patients.

\section{Materials and Methods}

\section{Patients and tissue samples}

The non-therapeutic intervened tumor and margin normal tissues were obtained after surgery from 55 GC patients in Ghaem Hospital of Mashhad University of Medical Sciences (MUMS). Trained interviewers obtained demographic and social habits of patients. The study was approved by ethics committee of MUMS. The resected tumor and normal tissues were histologically confirmed as GC and tumor free tissues, respectively. All the patients were new cases and had primary GC with no history of previous cancer in other organs. Furthermore, they did not receive any therapeutic plan such as chemotherapy or radiotherapy before the surgery. All the patients provided written informed consent. All dissected samples fixed in $70 \%$ formalin and embedded in paraffin.

\section{Immunohistochemistry}

Formalin fixed paraffin embedded (FFPE) sections with $3 \mu \mathrm{m}$ in thickness were prepared from gastric tumors and parallel normal specimens and loaded on poly-Llysine-coated slides for immunohistochemistry (IHC) study. Briefly, slides were dewaxed and rehydrated in a series of graded alcohol, placed in a retrieval solution named citrate buffer $10 \mathrm{mmol} / \mathrm{L}$ in $\mathrm{pH} 6.0$ to uncover the epitopes in a microwave oven for 40 minutes. The sections were allowed to cool down in room temperature and then incubated by endogenous peroxidases for 10 minutes to inactivate. Primary mouse monoclonal DPPA2 antibody (Abcam, Cambridge, UK) at a working dilution of $1 / 200$ at $4^{\circ} \mathrm{C}$ was used overnight followed by proceeded phosphate buffered saline washing. The primary antibody were detected with a secondary antibody, EnVi- sionTM+System/HRP, Rabbit/Mouse (DAB+) (DakoCy- tomation, Carpinteria, CA, USA). Decisively, staining results were visualized by Meyer's Hematoxylin, followed by acidified hematoxylin counter staining for 1 minute. Lastly, sections were steeped in a sequence of increasing concentrations of alcoholic solutions and washed up in xylene. DPPA2 positive section was used in every section analysis. Expert pathologists who were enchanted to the clinical and molecular results evaluated the tissue slides carefully.

\section{Evaluation of immunohistochemical staining results}

The intensity of immunohistochemical staining and the percentage of stained cells was assessed as previously described (Ghodsi et al.). The intensity of staining was considered as $1=$ weak, $2=$ moderate, and $3=$ strong . The percentage of positive stained cells was classified to five groups consisting of $0,<5 \% ; 1,5-25 \% ; 2,26-$ $50 \% ; 3,51-75 \%$; and $4,>75 \%$. Staining intensities then multiplied with the percentage of positive epithelial cells to generate the immunoreactivity score (IS) for each case. For example, if the staining intensity was strong (3) and the percentage of positive cells was $>80 \%$ (4), then the IS would be $3 \times 4=12$. Therefore, the IS ranged between 0 and 12 . While IS $=0$ was considered as negative, IS $<6$ and $\geq 6$ were considered as low and high level of protein expression, respectively.

\section{Statistical analysis}

Statistical analyses were performed by Statistical Package for the Social Sciences software version 16.0 (SPSS Inc., Chicago, IL, USA). Since the distribution of samples was normal, the correlations between DPPA2 protein expression and clinicopathological parameters were evaluated by Pearson test, independent sample t test and ANOVA. All tests were two-sided, and p values $<0.05$ were considered to be statistically significant.

\section{Results}

\section{Population study}

The population study was consisting of 35 males and 20 females with age ranged from 34 to 78 years at the time of diagnosis (mean \pm SD: $60.02 \pm 10.07$ ). The tumors size was between 1 and $12 \mathrm{~cm}$ with mean $( \pm \mathrm{SD})$ of 4.46 $( \pm 2.43)$. The tumor and their margin normal paraffin embedded GC samples were submitted to IHC-mediated analysis of the expression of DPPA2. Based on microscopy evaluations, all tumoral slides contained more than $70 \%$ tumor cells to ensure precise evaluation of gene expression in tumor tissues. At least five consecutive cross sections were analyzed for each tissue sample. Only cross sections of gastric tissues with markedly brown-stained cells, showing a clear structure, were scored positive for DPPA2 protein expression with nuclear localization.

Immunohistochemical Detection of DPPA2 Protein in GC Samples

Based on immunoreactivity results, DPPA2 was over 
Table 1. Correlations between DPPA2 Protein Expression and Clinicopathological Features of GC Patients

\begin{tabular}{|c|c|c|c|c|}
\hline \multirow{2}{*}{\multicolumn{2}{|c|}{ Clinicopathological variable }} & \multicolumn{2}{|c|}{$\begin{array}{l}\text { DPPA2 Expression } \\
\text { (Tumor) }\end{array}$} & \multirow[t]{2}{*}{$P$ value } \\
\hline & & Low & High & \\
\hline \multirow{2}{*}{ Sex } & Male & 18 & 17 & \multirow{2}{*}{0.674} \\
\hline & Female & 14 & 6 & \\
\hline \multirow{2}{*}{ Age } & $<50$ & 5 & 3 & \multirow{2}{*}{0.276} \\
\hline & $\geq 50$ & 27 & 20 & \\
\hline \multirow{2}{*}{$\begin{array}{l}\text { Lymph node } \\
\text { Metastasis }\end{array}$} & No & 10 & 7 & \multirow{2}{*}{$0.012^{*}$} \\
\hline & N1-3 & 22 & 16 & \\
\hline \multirow{4}{*}{$\begin{array}{l}\text { Depth of tumor } \\
\text { invasion }\end{array}$} & $\mathrm{T} 1$ & 1 & 1 & \multirow{4}{*}{0.831} \\
\hline & $\mathrm{T} 2$ & 5 & 3 & \\
\hline & T3 & 22 & 16 & \\
\hline & $\mathrm{T} 4$ & 4 & 3 & \\
\hline \multirow{6}{*}{$\begin{array}{l}\text { Stage of tumor } \\
\text { progression }\end{array}$} & I & 4 & 1 & \multirow{6}{*}{0.396} \\
\hline & IIA & 6 & 8 & \\
\hline & IIIA & 2 & 1 & \\
\hline & IIIB & 7 & 3 & \\
\hline & IIIC & 11 & 9 & \\
\hline & IV & 2 & 1 & \\
\hline \multirow{3}{*}{$\begin{array}{l}\text { Grade of tumor } \\
\text { differentiation }\end{array}$} & I & 6 & 8 & \multirow{3}{*}{0.211} \\
\hline & II & 12 & 7 & \\
\hline & III & 14 & 8 & \\
\hline
\end{tabular}

*Statistically significant

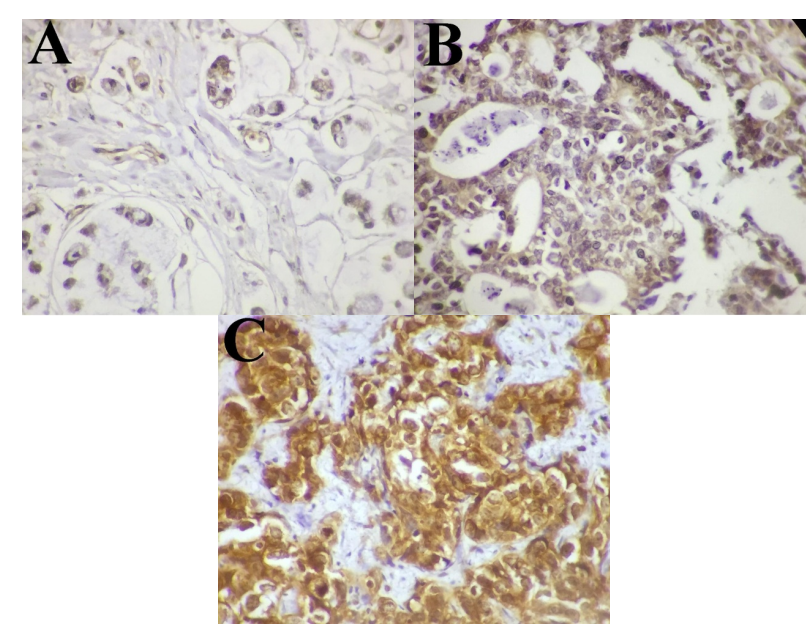

Figure 1. Expression of DPPA2 Protein in Gastric Cancer Tissues. A) Weak expression of DPPA2 in nearly $20 \%$ of the cells, B) Moderately expression of DPPA2 in nearly $90 \%$ of the cells, and C) Strong expression of DPPA2 in nearly $90 \%$ of the cells

expressed in GC cases compared with normal tissues (P $<0.005)$. While DPPA2 was expressed in all GC samples, its high expression was found in 23 of 55 tumor tissues (41.8\%). Interestingly, 50 of 55 normal samples (90.9\%) were negative for DPPA2 protein expression and remained 5 samples showed very low expression of DPPA2. DPPA2 protein expression in GC was significantly correlated with lymph node metastasis $(\mathrm{p}=0.012)$. These results showed that high DPPA2 expression was associated with aggressiveness of gastric cancer. The clinicopathological features of the patients are presented in Table 1. Different levels of DPPA2 protein expression in GC tissues are showed in Figure 1.

\section{Discussion}

Deprogramming as the center point of stem cell theory governs differentiated cell behavior through returning the cell to the proliferative, undifferentiated, stem cell state (Monk et al., 1987; Mayer et al., 2000; Reik et al., 2001). Blastocyst inner cell mass and other embryonic stem cells, as totipotent stem cells, are capable to give rise to the immortal cell lines in vitro and teratomas in vivo. Active embryonic genes at this stage may be associated with similar properties of deprogramming, maintenance of the undifferentiated cell state, proliferation, invasiveness, and indefinite growth of cancer cells (John et al., 2008). DPPA2 as an embryo-cancer transcript is expressed only in pluripotent stem cells and developing germ line cells, and is absent in normal differentiated somatic cells, but re-expressed in tumor tissue (Monk and Holding, 2001). To explore the probable role of DPPA2 in GC progression and development, its expression was studied in GC tumors compared to their adjacent normal tissues. The results demonstrated significant increase in DPPA2 expression in gastric dysplasia in comparison with normal gastric tissues. The clinical relevance of DPPA2 in GC illustrated that high level expression of this protein was associated with lymph node metastasis supporting this hypothesis that alteration in DPPA2 expression may be an early event in gastric carcinoma. The newly identified members of pluripotency related oncogenes consisting of DPPA2 and DPPA4 have different binding sites for POU domain and SOX proteins which demonstrates the important role of these proteins in regulatory pathways of maintaining stem cells pluripotency (Monk et al., 2008). SAP domain of the DPPA2 protein plays important role in chromatin organization and RNA processing. DPPA2 is correlated to a closely linked gene, DPPA4, on human chromosome 3 (Gagliardi et al., 2013). It has been reported that there are similarities between DPPA2 and DPPA4 (Tung et al., 2013). DPPA4 is highly expressed in embryonal carcinomas, pluripotent germ cell tumors, and other malignancies such as bladder, prostate, and colon cancers (Tung et al., 2013; Amini et al., 2014). Over expression of DPPA4 generates oncogenic foci in sarcoma cells and causes independent growth (Tung et al., 2013). In addition, DPPA4 over expression induces cell proliferation through genes related to regulation of G1/S transition (Tung et al., 2013). On the other hand down-regulation of DPPA4 in E14 mouse embryonic stem cells and P19 mouse embryonic carcinoma cells causes decreased cell proliferation in both cases (Tung et al., 2013). Furthermore, functional analysis indicated that both the DNA-binding SAP domain and the histone-binding C-terminal domain of the protein are significantly crucial for the oncogenic transformation activity of DPPA4 (Tung et al., 2013). Although little is known about DPPA2, its expression has been reported in sparse cancers. DPPA2 
is over expressed in colorectal cancer with significant correlation to the lymph node metastasis of tumor cells in advanced stages (III/IV) of the tumor development (Raeisossadati et al., 2014; Ghodsi et al., 2015). It is also expressed in lung and liver cancers (John et al., 2008; Monk et al., 2008). Moreover, expression of DPPA2 as a human cancer antigen was demonstrated in $30 \%$ of nonsmall cell lung carcinomas as well as a small percentages of melanomas, lymphomas, colorectal and ovarian cancers (John et al., 2008). These findings may indicate a shared function for DPPA2 in embryonic stem cells and cancer stem cells showing that some functions in embryogenesis, specifically restricted to the stage of the emergence and/ or maintenance of the embryonic stem cell, are generally involved in the cancer stem cell phenotype (Monk et al., 2008). Probably there is a close relationship between DPPA 2 and pluripotency genes regulatory network consisting of OCT4, SOX2, NANOG, SALL4, and DPPA4 (Yang et al., 2008; Gagliardi et al., 2013; Forghanifard et al., 2014). Furthermore, it has been illustrated that Dppa4, as well as components of the pluripotency regulatory network, is required for self-renewal, interestingly (Ivanova et al., 2006). The similarity between the function of DPPA4 and DPPA2 in self-renewal and pluripotency of ES cells may indicates that over expression of DPPA2 in cancer cells imitates similar roles. As a consequent, DPPA2 may play dominant roles in embryonic stem cells and supposedly in cancer stem cells with different probable protein-DNA and protein-protein interactions with strategic regulatory factors involved in maintenance of stemness state, self-renewal and pluripotency of the cell. Over expression of DPPA2 may be caused through different molecular mechanisms in GC. However, the most probable root of DPPA2 over expression may be through regulatory network of pluripotency and stemness state of the cell consisting of master regulators OCT4, SOX2 and NANOG. There are different evidences shedding light in this way. OCT4 and SOX2 are not only associated with induced pluripotent stem cells, but they have also oncogenic behavior in different malignancies suggesting a correlation between these stemness factors and cancer stem cells (Matsuoka et al., 2012). It has been demonstrated that OCT4 over expression was not only correlated with invasiveness of GC, but also was associated with GC tumor differentiation (Huang et al., 2012; Kong et al., 2014). Hütz et al indicated that SOX2 governs several prospects of GC development and progression by regulating the expression of members of important signaling pathways (Hutz et al., 2014). SOX2positive expression might be associated with invasiveness of gastric cancer (Matsuoka et al., 2012). NANOG is also closely associated with cell proliferation, the cell cycle, apoptosis, migration and invasion abilities of gastric cancer cells (Ji and Jiang, 2013). Since, SOX2, OCT4 and NANOG as stemness factors are over expressed in GC, and according to the function of DPPA2 along with OCT4 and NANOG in the ES cells (Maldonado-Saldivia et al., 2007; Monk et al., 2008), we supposedly hypothesize that over expression of DPPA 2 may be caused by pluripotency regulatory network consisting of OCT4, SOX2 and NANOG through direct or indirect mechanisms. In addition, aberrant induction of intestinal-specific homeobox transcription factors, CDX1 and CDX2, by Helicobacter pylori has important roles in modifications of stemness factors SOX2, OCT3/4 and NANOG to generate cancer stem cells. CDX1 also activate directly SALL4 and KLF5 as stemness-related reprogramming factors in GC (Akhavan-Niaki and Samadani, 2014). Therefore, Helicobacter pylori infection may also cause expression of major pluripotency and stemness state regulators SALL4, KLF4, OCT3/4, SOX 2 and NANOG through activation of CDX1 and CDX2 leading to over expression of DPPA2.

In conclusion, we showed that DPPA2 is expressed in GC in significant correlation with aggressiveness of the disease. To the best of our knowledge, this is the first report focusing on clinicopathological relevance of DPPA2 in GC. DPPA2 may be introduced as a new marker for invasive and metastatic GCs.

\section{Acknowledgements}

The authors gratefully acknowledge colleagues at Department of Pathology, Ghaem Hospital, Mashhad University of Medical Sciences, for their assistance in sample preparation.

\section{References}

Akhavan-Niaki H, Samadani AA (2014). Molecular insight in gastric cancer induction: an overview of cancer stemness genes. Cell Biochem Biophys, 68, 463-73.

Amini S, Fathi F, Mobalegi J, et al (2014). The expressions of stem cell markers: Oct4, NANOG, SOX2, nucleostemin, Bmi, Zfx, Tcl1, Tbx3, Dppa4, and Esrrb in bladder, colon, and prostate cancer, and certain cancer cell lines. Anat Cell Biol, 47, 1-11.

Aravind L, Koonin EV (2000). SAP - a putative DNA-binding motif involved in chromosomal organization. Trends Biochem Sci, 25, 112-4.

Cho J, Lim J, Cheong J, et al (2011). Gene expression signaturebased prognostic risk score in gastric cancer. Clin Cancer Res, 17, 1850-7.

Du J, Chen T, Zou X, et al (2010). Dppa2 knockdown-induced differentiation and repressed proliferation of mouse embryonic stem cells. J Biochem, 147, 265-71.

Ferlay J, Parkin DM, Steliarova-Foucher E (2010). Estimates of cancer incidence and mortality in Europe in 2008. Eur J Cancer, 46, 765-81.

Forghanifard MM, Ardalan Khales S, Javdani-Mallak A, et al (2014). Stemness state regulators SALL4 and SOX2 are involved in progression and invasiveness of esophageal squamous cell carcinoma. Med Oncol, 31, 922.

Gagliardi A, Mullin NP, Ying Tan Z, et al (2013). A direct physical interaction between NANOG and SOX2 regulates embryonic stem cell self-renewal. EMBO J, 32, 2231-47.

Ghodsi M, Jafarian AH, Montazer M, et al (2015). Diagnostic clinical relevance of developmental pluripotency-associated 2 (DPPA2) in colorectal cancer. Int J Surg.

Huang ZJ, Wang R, Luo WY, et al (2012). Expression of OCT4 in gastric cancer cell lines and its significance. Sichuan $D a$ Хие Хие Bao Yi Хие Ban, 43, 812-5.

Hudler P (2012). Genetic aspects of gastric cancer instability. Scientific World J, 2012, 761909.

Hutz K, Mejias-Luque R, Farsakova K, et al (2014). The stem cell factor SOX2 regulates the tumorigenic potential in human 
gastric cancer cells. Carcinogenesis, 35, 942-50.

Ivanova N, Dobrin R, Lu R, et al (2006). Dissecting self-renewal in stem cells with RNA interference. Nature, 442, 533-8.

Ji W, Jiang Z (2013). Effect of shRNA-mediated inhibition of NANOG gene expression on the behavior of human gastric cancer cells. Oncol Lett, 6, 367-74.

John T, Caballero OL, Svobodova SJ, et al (2008). ECSA/DPPA2 is an embryo-cancer antigen that is coexpressed with cancertestis antigens in non-small cell lung cancer. Clin Cancer Res, 14, 3291-8.

Kong D, Su G, Zha L, et al (2014). Coexpression of HMGA2 and Oct4 predicts an unfavorable prognosis in human gastric cancer. Med Oncol, 31, 130.

Maldonado-Saldivia J, van den Bergen J, Krouskos M, et al (2007). Dppa2 and Dppa4 are closely linked SAP motif genes restricted to pluripotent cells and the germ line. Stem Cells, 25, 19-28.

Matsuoka J, Yashiro M, Sakurai K, et al (2012). Role of the stemness factors SOX2, oct $3 / 4$, and NANOG in gastric carcinoma. J Surg Res, 174, 130-5.

Mayer W, Niveleau A, Walter J, et al (2000). Demethylation of the zygotic paternal genome. Nature, 403, 501-2.

Monk M, Boubelik M, Lehnert S (1987). Temporal and regional changes in DNA methylation in the embryonic, extraembryonic and germ cell lineages during mouse embryo development. Development, 99, 371-82.

Monk M, Hitchins M, Hawes S (2008). Differential expression of the embryo/cancer gene ECSA(DPPA2), the cancer/testis gene BORIS and the pluripotency structural gene OCT4, in human preimplantation development. Mol Hum Reprod, 14, 347-55.

Monk M, Holding C (2001). Human embryonic genes reexpressed in cancer cells. Oncogene, 20, 8085-91.

Mottaghi-Dastjerdi N, Soltany-Rezaee-Rad M, Sepehrizadeh $\mathrm{Z}$, et al (2014). Genome expression analysis by suppression subtractive hybridization identified overexpression of Humanin, a target gene in gastric cancer chemoresistance. Daru, 22, 14.

Raeisossadati R, Abbaszadegan MR, Moghbeli M, et al (2014). Aberrant expression of DPPA2 and HIWI genes in colorectal cancer and their impacts on poor prognosis. Tumour Biol.

Reik W, Dean W, Walter J (2001). Epigenetic reprogramming in mammalian development. Science, 293, 1089-93.

Takeno A, Takemasa I, Seno S, et al (2010). Gene expression profile prospectively predicts peritoneal relapse after curative surgery of gastric cancer. Ann Surg Oncol, 17, 1033-42.

Tan IB, Ng I, Tai WM, et al (2012). Understanding the genetic basis of gastric cancer: recent advances. Expert Rev Gastroenterol Hepatol, 6, 335-41.

Tchabo NE, Mhawech-Fauceglia P, Caballero OL, et al (2009). Expression and serum immunoreactivity of developmentally restricted differentiation antigens in epithelial ovarian cancer. Cancer Immun, 9, 6.

Tung PY, Varlakhanova NV, Knoepfler PS (2013). Identification of DPPA4 and DPPA2 as a novel family of pluripotencyrelated oncogenes. Stem Cells, 31, 2330-42.

Wu JY, Cheng CC, Wang JY, et al (2014). Discovery of tumor markers for gastric cancer by proteomics. PLoS One, 9 , 84158.

Yang J, Chai L, Fowles TC, et al (2008). Genome-wide analysis reveals Sall4 to be a major regulator of pluripotency in murine-embryonic stem cells. Proc Natl Acad Sci U S A, 105, 19756-61.

Zabaleta J (2012). Multifactorial etiology of gastric cancer. Methods Mol Biol, 863, 411-35. 